\title{
Low-extinction windows in the inner Galactic Bulge
}

\author{
C. M. Dutra ${ }^{1,2}$, B. X. Santiago ${ }^{1}$, and E. Bica ${ }^{1}$ \\ 1 Universidade Federal do Rio Grande do Sul, IF, CP 15051, Porto Alegre 91501-970, RS, Brazil \\ 2 Instituto Astronomico e Geofisico da USP, CP 3386, São Paulo 01060-970, SP, Brazil
}

Received 9 March 2001 / Accepted 30 October 2001

\begin{abstract}
We built $K$ band extinction maps in the area of two candidate low-extinction windows in the inner Bulge: W0.2-2.1 at $(\ell, b)=\left(0.25^{\circ},-2.15^{\circ}\right)$, and W359.4-3.1 at $(\ell, b)=\left(359.40^{\circ},-3.10^{\circ}\right)$. We employed $J H K_{\mathrm{s}}$ photometry from the 2MASS Point Source Catalog. Extinction values were determined by fitting the upper giant branch found in the present 2MASS $K_{\mathrm{s}}\left(J-K_{\mathrm{s}}\right)$ diagrams to a de-reddened bulge stellar population reference giant branch. We tested the method on the well known Baade's and Sgr I windows: the 2MASS mean extinction values in these fields agreed well with those of previous studies. We confirm the existence of low-extinction windows in the regions studied, as local minima in the $A_{K}$ maps reaching $A_{K}$ values about 2 standard deviations below the mean values found in the neighbouring areas. Schlegel et al.'s (1998) FIR extinction maps, which integrate dust contributions throughout the Galaxy, are structurally similar to those derived with 2MASS photometry in the two studied windows. We thus conclude that the dust clouds affecting the 2MASS and FIR maps in these directions are basically the same and are located on foreground of the bulk of bulge stars. However, the $A_{K}$ absolute values differ significantly. In particular, the FIR extinction values for W359.4-3.1 are a factor $\simeq 1.45$ larger than those derived from the 2MASS photometry. Possible explanations of this effect are discussed. The lower Galactic latitudes of the low-extinction windows W359.4-3.1 and W0.2-2.1, as compared to Baade's Window, make them promising targets for detailed studies of more central bulge regions.
\end{abstract}

Key words. Galaxy: interstellar medium: dust

\section{Introduction}

Most of the Bulge stellar population is still largely unstudied due to the combined effects of large distances and high extinction. The information on Bulge stellar populations comes mainly from either globular clusters or from its $\mathrm{M}$ and $\mathrm{K}$ field giant stars. Yet, the study of the age and metallicity distribution in this region is of considerable importance, since a comprehensive study of the resolved Galactic Bulge allows us to better understand the bulge of external early and late type galaxies. Furthermore, inferring the main properties of the Bulge and comparing them to those of other components of the Galaxy is likely to provide clues to unveiling the process of galaxy formation (Aguerri et al. 2001; Wyse et al. 2000).

During the last years the Galactic Bulge stellar population has been studied mainly in the direction of lowextinction regions (Lloyd Evans 1976; Whitford 1978;

Send offprint requests to: B. X. Santiago,

e-mail: santiago@if.ufrgs.br
Terndrup 1988; Tiede et al. 1995; Alard et al. 2001). Baade (1963) identified the windows Sgr I, Sgr II, and the NGC 6522 field; this latter has been widely referred to as Baade's Window in subsequent studies. Stanek (1996) studied the extinction distribution in Baade's Window using the OGLE photometry of red clump stars, obtaining values from $A_{V}=1.26$ up to $A_{V}=2.79$. Frogel et al. (1999, hereafter FTK99) determined the extinction for 11 inner Bulge fields using Baade's Window red giant branch as a reference, yielding values in the range $A_{V}=2.41$ up to 19.20 .

Recently, wide-angle near infrared (NIR) surveys such as the Two Micron All Sky Survey (hereafter, 2MASS; Skrutskie et al. 1997) and the Deep NIR Southern Sky Survey (DENIS; Epchtein et al. 1997) have allowed investigations of the stellar population (Unavane et al. 1998) and reddening (Schultheis et al. 1999) in the inner Bulge. Schultheis et al. (1999) mapped the extinction in the inner Bulge for $|\ell|<8^{\circ}$ and $|b|<1.5^{\circ}$ (with a resolution of $4^{\prime}$ ) using isochrone fitting to the colour-magnitude 
diagrams (CMDs) obtained from DENIS $J, K_{\mathrm{S}}$ observations. The extinction varies from $A_{V} \approx 6$ up to $A_{V} \approx 37$. They showed that the extinction and, as a consequence, the dust clouds in the inner Bulge, present a very patchy distribution.

In the present study we use the 2MASS survey in the $J$ $(1.25 \mu \mathrm{m}), H(1.65 \mu \mathrm{m})$ and $K_{\mathrm{s}}(2.17 \mu \mathrm{m})$ bands to identify low-extinction windows in the inner Galactic Bulge. In Sect. 2 we discuss the process to select low-extinction candidate regions in the inner Galactic Bulge. In Sect. 3 we discuss the method of deriving extinction values throughout these regions, provide a reddening distribution map for them and analyse the results. Finally, the concluding remarks are given in Sect. 4.

\section{Selecting low-extinction windows}

In search for candidate low-extinction regions we used the DIRBE/IRAS dust emission redddening map from Schlegel et al. (1998, hereafter SFD98), which is available in Web Interface http://astro.berkeley.edu/dust. The extinction maps derived from these far infra-red observations will be referred to as "FIR extinction maps" throughout the present work. Analogously, we will use the notation $A_{K, F I R}$ to denote the $K$ band extinction derived from SFD98 data. Stanek (1998) identified two low-extinction windows on the FIR extinction maps, centred at $(\ell, b)=\left(0^{\circ},-2^{\circ}\right)$ and $(\ell, b)=\left(4^{\circ},-3^{\circ}\right)$. Although the FIR extinction corresponds to the contribution of the entire dust column throughout the Galaxy (Dutra \& Bica 2000), its angular distribution can help with the selection of potentially interesting areas of low-extinction towards the Bulge. We obtained $E(B-V)_{\mathrm{FIR}}$ values in the field of $10^{\circ} \times 10^{\circ}$ around the Galactic Centre from the original overall FIR map in SFD98, using an extraction tool thereby provided. Figure 1 shows the resulting $A_{K, F I R}$ extinction map, where the positions of the three lowreddening regions from Baade (1963), two from Stanek (1998), and a newly identified one, are indicated. The transformation from $E(B-V)_{\mathrm{FIR}}$ to $A_{K, F I R}$ assumed $A_{K}=0.112 A_{V}$ and $R_{V}=A_{V} / E(B-V)=3.1$ (Cardelli et al. 1989).

In Table 1 we condense the basic information on the mentioned bulge windows. By columns: (1) adopted designation throughout the present study (a practical way to refer to the windows is "W" followed by its values of galactic coordinates), (2) other designations, (3) and (4) galactic coordinates, (5) approximate angular dimensions, (6) reference for window identification, and (7) extinction map and photometric source.

\section{Extinction within the candidate windows}

\subsection{FIR extinction maps}

Figure 2 shows the $A_{K}$, FIR maps within $2^{\circ} \times 2^{\circ}$ around $(\ell, b)=\left(0^{\circ},-2^{\circ}\right)$. This region should contain W0.22.1 , listed in Table 1 and which was originally identified

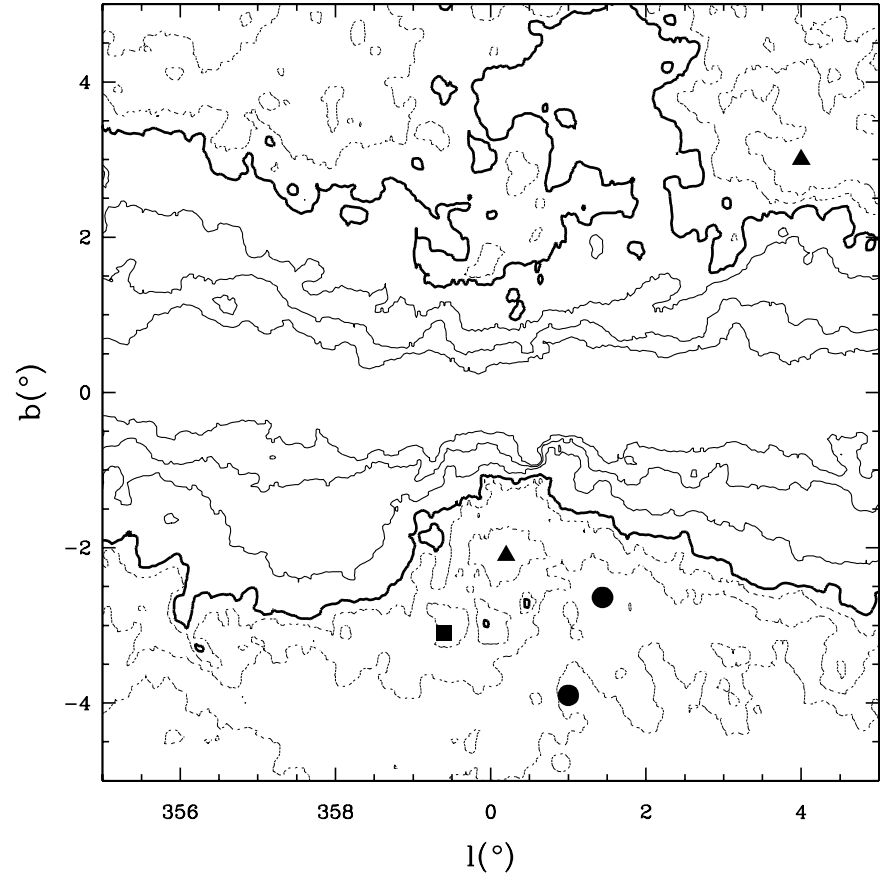

Fig. 1. $10^{\circ} \times 10^{\circ}$ FIR extinction map centred on the Galactic Nucleus. The line width is coded according to the $A_{K, F I R}$ value. Dotted lines correspond to lower than average levels: $0.7,1.05$ and 1.4. The thick solid line is close to the field average and corresponds to $A_{K, F I R}=1.75$. The thin solid lines are above average: $3,6,10$. Filled circles indicate positions of windows from Baade (1963), triangles from Stanek (1998), and the square from the present study.

by Stanek (1998). In fact, we can more precisely locate this window at $(\ell, b)=\left(0.25^{\circ},-2.15^{\circ}\right)$, with dimensions $\approx 60^{\prime} \times 40^{\prime}$. This is a nearly closed region in Fig. 2 where $A_{K}$, FIR values are systematically below the average value in the map, $<A_{K}$, FIR $>=0.41$. The lowest $A_{K}$, FIR values within W0.2-2.1 reach down to $A_{K}$, FIR $=0.28$, which corresponds to about 2 standard deviations (std) below the mean. Therefore, there is a well defined local $A_{K}$, FIR minimum in this area.

Figure 3 shows a similar map centred at $(\ell, b)=$ $\left(0^{\circ},-2^{\circ}\right)$, close to the new candidate low-extinction region proposed in this work, W359.4-3.1. A distinct region with lower than average $A_{K}$, FIR values is again visible; it is centred at $(\ell, b)=\left(359.40^{\circ},-3.10^{\circ}\right)$, with $40^{\prime} \times 30^{\prime}$ in size. This region has a deeper minimum, reaching $A_{K}$, FIR $=0.24$, which is 2.5 std below the average map value of $\left\langle A_{K}\right.$, FIR $>=0.42$. It is also more circular in shape and completely encircled by areas of larger $A_{K}$, FIR values. We should point out that, by inspection of the FIR extincion map in Fig. 1, one notices that even the average $A_{K}$, FIR values in Figs. 2 and 3 are very atypical for their $(\ell, b)$ location.

\subsection{Deriving $A_{K}$ from $2 M A S S$ data}

An alternative way to obtain $K$ band extinction values and to build extinction maps for W0.2-2.1 and 
Table 1. Low-extinction windows towards central parts of the Galaxy.

\begin{tabular}{lcccccc}
\hline \hline Name & Other Designation & $\ell\left(^{\circ}\right)$ & $b\left(^{\circ}\right)$ & $d\left(^{\prime}\right)$ & Reference & Photometry \\
\hline W359.4-3.1 & - & 359.40 & -3.10 & $40 \times 30$ & present study & 2MASS (present study) \\
W0.2-2.1 & $(l, b)=(0,-2)$ Window & 0.24 & -2.14 & $60 \times 40$ & Stanek (1998) & 2MASS (present study) \\
Baade's Window & NGC6522 field,W1.0-3.9 & 1.04 & -3.88 & 60 & Baade (1963) & OGLE (Stanek 1996) \\
Sgr I Window & W1.4-2.6 & 1.44 & -2.64 & 60 & Baade (1963) & - \\
W4.0+3.0 & $(l, b)=(4,3)$ Window & 4.00 & 3.00 & $180 \times 100$ & Stanek (1998) & - \\
Sgr II Window & W4.2-5.1 & 4.15 & -5.14 & 85 & Baade (1963) & - \\
\hline
\end{tabular}

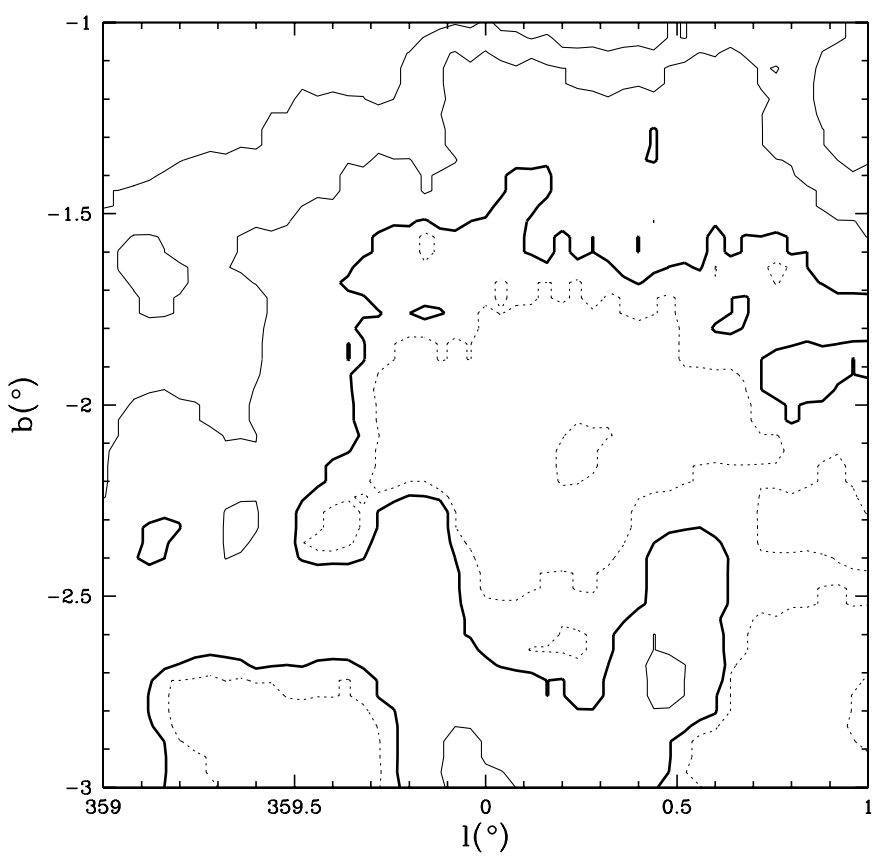

Fig. 2. FIR extinction map of window W0.2-2.1. Contour levels correspond to $A_{K, \text { FIR }}=0.29$ and 0.37 (dotted lines), 0.41 (thick solid line), and 0.57 and 0.83 (thin solid lines).

W359.4-3.1 is to use the 2MASS $J K_{\mathrm{S}}$ photometry available in Web Interface

http://irsa.ipac.caltech.edu/applications/ Gator/.

2MASS also provides $H$ band data, but we prefer not to use them, since the $\left(J-K_{\mathrm{s}}\right)$ colours appear to be more sensitive to extinction variations than the $\left(H-K_{\mathrm{s}}\right)$ ones; this is probably due to the larger wavelength interval.

We extracted the 2MASS data for stars with $8.0 \leq$ $K_{\mathrm{S}} \leq 11.5$ within $1^{\circ}$ radius centred on the Galactic coordinates $(\ell, b)=\left(0.0^{\circ},-2.0^{\circ}\right)$ and $(\ell, b)=\left(359.0^{\circ}\right.$, $\left.-3.0^{\circ}\right)$. These are the same centers as in the FIR extinction maps discussed in the previous subsection. The choice of $K_{\mathrm{s}}$ magnitude range for data extraction is motivated by the fact that the upper giant branch is as well defined and linear in this range as in Baade's window. The total number of extracted stars from the 2MASS database were 90407 in the W0.2-2.1 region and 69286 in the W359.43.1 region. For comparison, we also extracted 2MASS data from regions at $(\ell, b)=\left(1.0^{\circ},-3.0^{\circ}\right)$ and $(\ell, b)=$ $\left(1.0^{\circ},-4.0^{\circ}\right)$, which include the Sgr I (48040 stars) and Baade's (20 358 stars) windows, respectively (see Table 1).

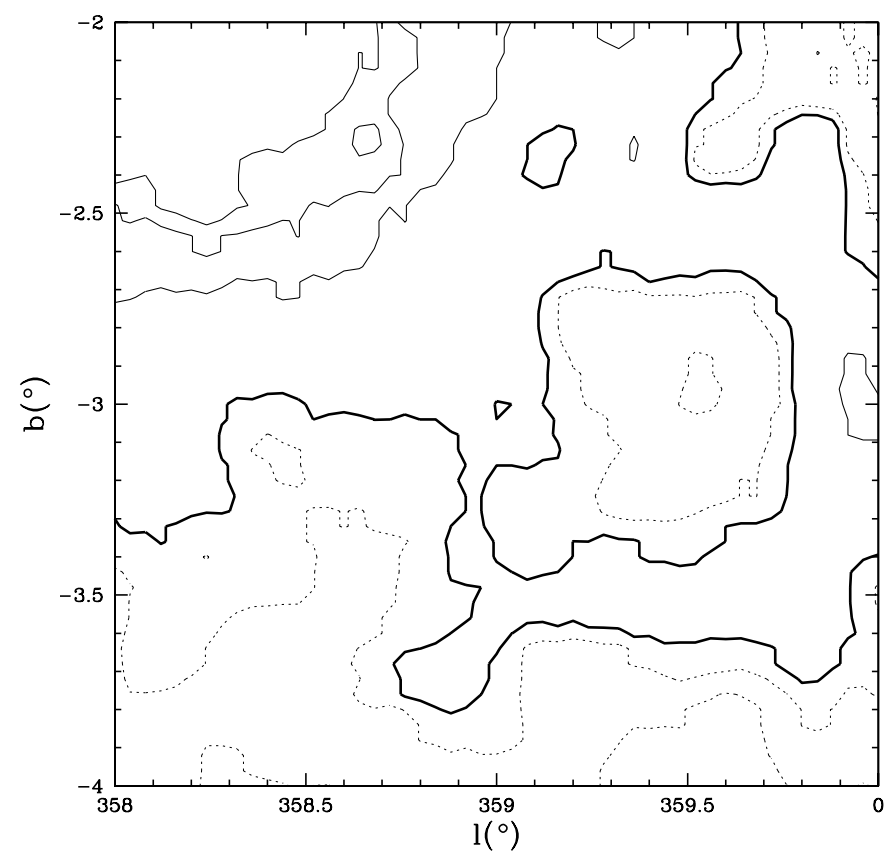

Fig. 3. FIR extinction map of window W359.4-3.1. The contour levels correspond to $A_{K}$, FIR $=0.31$ and 0.38 (dotted lines), 0.42 (thick solid line), and $0.59,0.76$ and 0.87 (thin solid lines).

We note, however, that the 2MASS archive data do not yet provide complete coverage of these two comparison fields. The mean magnitude errors from the extracted 2MASS data are $\langle\sigma\rangle_{J}=0.04 \pm 0.01$ and $\langle\sigma\rangle_{K_{\mathrm{S}}}=0.04 \pm$ 0.01. These photometric errors bracket $95 \%$ and $92 \%$ of all extracted stars, respectively for $J$ and $K_{\mathrm{S}}$.

In order to map the selected low-extinction regions in $A_{K}$, we define small square cells with $4^{\prime} \times 4^{\prime}$. The extinction in each cell was determined by upper giant branch fitting to its observed CMD, similar to the FTK99 extinction determination method. FTK99 derived $A_{K}$ values for their fields using the upper giant branch of Baade's window (Tiede et al. 1995) as reference. Our reference upper giant branch was defined from that of FTK99. We proceeded as follows: we first extracted 2MASS photometry for seven fields from FTK99. These fields are g0-1.8a, g0-2.3a, g0-2.8a, g1-1.3a, g2-1.3a, g3-1.3a and g4-1.3a, where the field designation incorporates its position in Galactic coordinates. We used the $K$ and $K_{\mathrm{s}}$ filter transmission curves given in Persson et al. (1998) and the extinction curve of Cardelli et al. (1989) to obtain the 


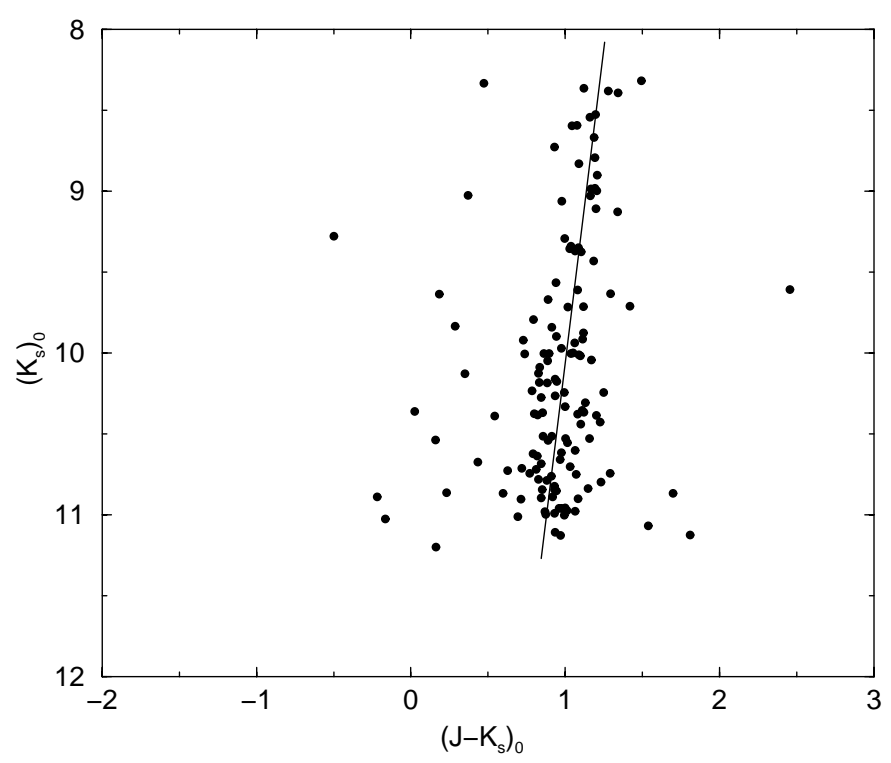

Fig. 4. Determination of the upper giant branch mean locus from the combined CMD of the fields in Frogel et al. (1999).

ratios $\frac{A_{K_{\mathrm{s}}}}{A_{V}}=0.118, \frac{A_{K}}{A_{V}}=0.112$ and $\frac{A_{K}}{A_{K_{\mathrm{S}}}}=0.95$. These ratios allow us to transform from $A_{K_{\mathrm{s}}}$ to $A_{K}$. The relation between extinction and reddening $A_{K}=0.618 E(J-K)$ (Mathis 1990) was used to derive the relation

$A_{K_{\mathrm{s}}}=0.670 E\left(J-K_{\mathrm{s}}\right)$.

We then extinction corrected the 2MASS CMDs of each of the 7 FTK99 fields and made a composite corrected CMD. Figure 4 shows the final CMD based on 2MASS data in FTK99's fields. It is clearly dominated by an upper giant branch, whose mean locus is shown as a straight line. This latter is the result of a linear fit to the upper giant branch in the adopted magnitude range, yielding

$$
\left(K_{\mathrm{S}}\right)_{0}=-7.81 *\left(J-K_{\mathrm{S}}\right)_{0}+17.83 .
$$

Assuming that the upper giant branch in each of our $4^{\prime} \times 4^{\prime}$ cells is comparable to and has the same slope as the extinction-corrected template given by Eq. (2), we can infer the extinction in each cell within the W0.2-2.1 and W359.4-3.1 regions. From the $\left(K_{\mathrm{s}}, J-K_{s}\right)$ CMD values of each star in the cell, we calculated the shift along the reddening vector given by Eq. (1) required to make it fall onto the reference upper giant branch. The $A_{K}$ value for each cell was taken as the median of the distribution of such values. Foreground contamination was minimized by applying a $2-\sigma$ clipping to the data and recalculating the median extinction and deviation iteratively until convergence. We hereafter use the notation $A_{K}$, 2MAss to denote the extinction value determined in this way.

In panels (5a) and $(5 \mathrm{c})$ we show extinction-corrected $\left(K_{\mathrm{s}}\right)_{0},\left(J-K_{\mathrm{s}}\right)_{0}$ CMDs for stars of typical cells in the W0.2-2.1 and W359.4-3.1 maps, respectively. Each CMD was corrected by the median extinction derived from the histograms of $A_{K}$ values in each cell. These histograms are shown in panels (5b) and (5d) for the cells in W0.2-2.1 and W359.4-3.1 maps, respectively, their median values being
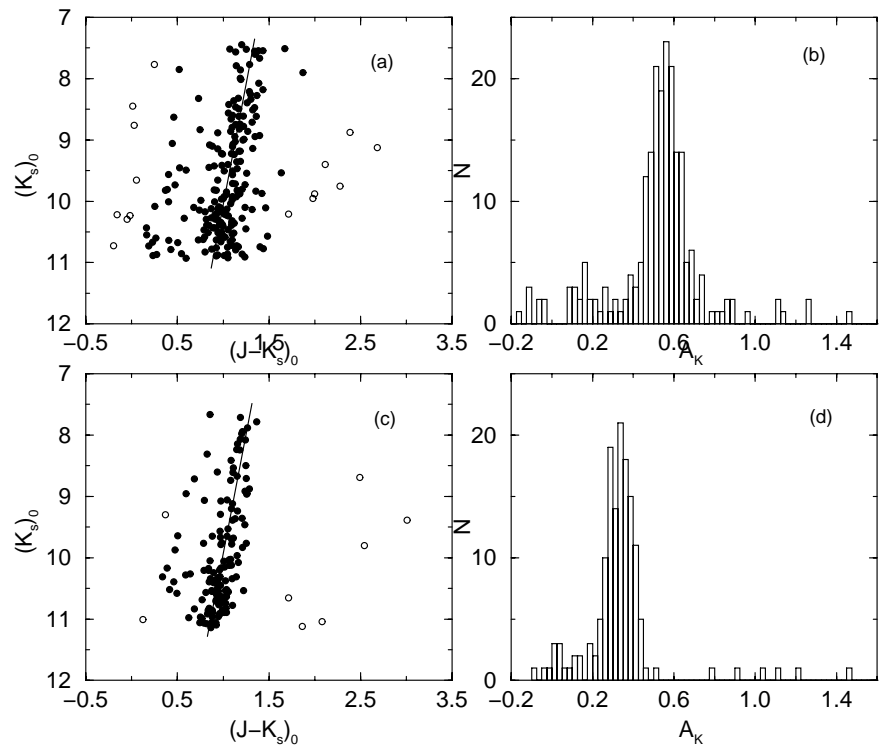

Fig. 5. a) $\left(K_{\mathrm{s}}\right)_{0},\left(J-K_{\mathrm{s}}\right)_{0} \mathrm{CMD}$ for the cell at $\ell=0.07^{\circ} b=$ $-1.2^{\circ}$ in the W0.2-2.1 field; b) $A_{K}$ histogram derived from $\mathrm{CMD}$ in a) as described in the text; c) $\left(K_{\mathrm{s}}\right)_{0},\left(J-K_{s}\right)_{0} \mathrm{CMD}$ for the cell at $\ell=359.2^{\circ} \quad b=-2.2^{\circ}$ in the W359.4-3.1 field; d) $A_{K}$ histogram derived from CMD in c) as described in text. The open circles in the CMDs indicate stars rejected due to the 2- $\sigma$ clipping of the $A_{K}$ distribution. The solid lines in the CMDs indicate the reference upper giant branch locus (Eq. (2)).

$A_{K, 2 \mathrm{MASS}}=0.54 \pm 0.16$ and $A_{K, 2 \mathrm{MASS}}=0.33 \pm 0.10$. We note that there are secondary upper giant branches in the CMDs and consequently a second peak in $A_{K}$ distribution for the two cells (at $\left\langle A_{K}\right\rangle=0.14$ and 0.02 for the chosen cells in W0.2-2.1 and W359.4-3.1, respectively). These are probably caused by a secondary dust layer or to a dust cloud variation scale smaller than 4 arcmin along the lines of sight considered.

For the comparison regions, which include the lowextinction Sgr I and Baade's windows, we obtain $A_{K, 2 \mathrm{MASS}}=0.23 \pm 0.05$ and $A_{K, 2 \mathrm{MASS}}=0.18 \pm 0.04$, respectively, using the same method described above. Glass et al. (1995) studied variable stars in the Sgr I window and adopted $A_{K}=0.21$. For Baade's Window, considering the extinction map obtained by Stanek (1996) and zero-point calibrations (Gould et al. 1998; Alcock et al. 1998), the mean extinction is $\left\langle A_{K}\right\rangle=0.17 \pm 0.03$. The adopted extinction determination method and the 2MASS photometry produce results compatible to those found in the literature. Thus, they are certainly useful to map the extinction in the inner Galactic Bulge, although one has to keep in mind that regions much closer to the Galactic Centre are more affected by crowding and highly variable extinction, which may in turn have an impact on photometric precision and on the applicability of the method. Another issue is the possible existence of metallicity gradients in the stellar content of the inner Bulge, which may result in systematic errors in the inferred extinction values (Schultheis et al. 1999). However, there 


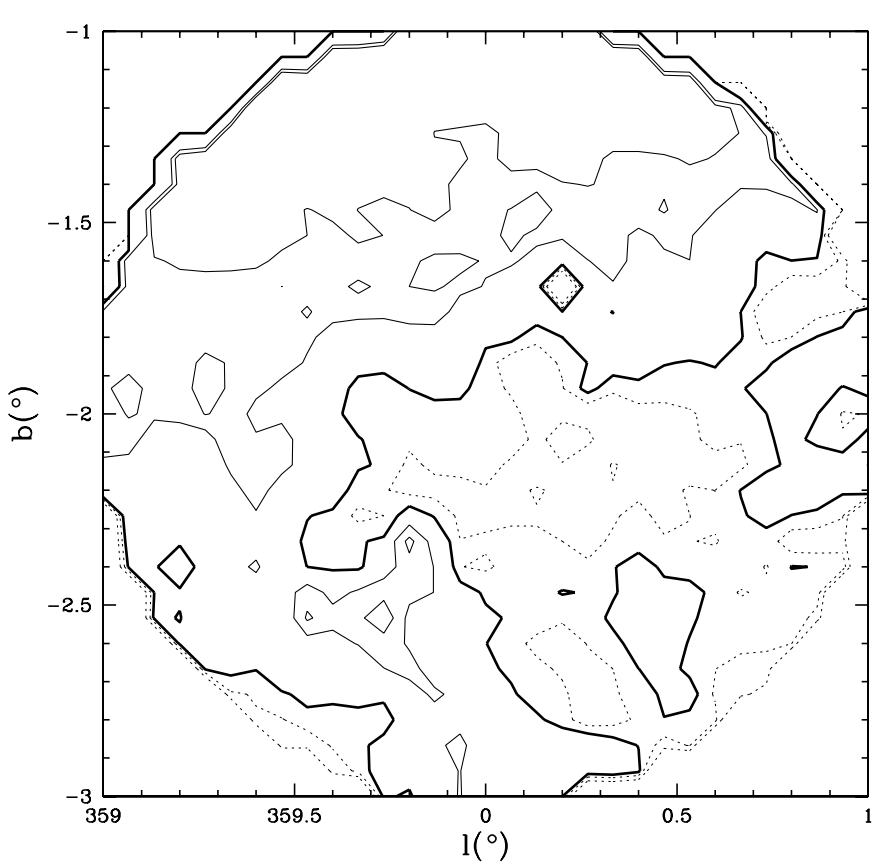

Fig. 6. 2MASS extinction map of window W0.2-2.1. Contour levels correspond to $A_{K}$, 2MASS $=0.2$ and 0.25 (dotted lines), 0.29 (thick solid line), and 0.4 and 0.5 (thin solid lines).

is some recent evidence, based on spectroscopy of M giants, against significant metallicity variations in that region (Ramírez et al. 2000).

\subsection{MASS extinction maps}

Figures 6 and 7 show the $A_{K,}$ MASS maps for the W0.22.1 and W359.4-3.1 regions, respectively. These contour maps are similar in shape to those shown in Figs. 2 and 3 . In both figures we see nearly closed regions with $0.2<A_{K, 2 \mathrm{MASS}}<0.25$. The mean extinction values are $<A_{K}$, 2MASS $>=0.29 \pm 0.05$ in W0.2-2.1 and $<A_{K}$, MASS $>=0.28 \pm 0.04$ in W359.4-3.1. Therefore, some cells within the low-extinction windows have $A_{K}$, 2MAss value about 2 std below the mean map values. Note that the lack of contours in the corners of the figures just reflects the circular areas used for the 2MASS extractions.

Panels a) and c) of Fig. 8 show the histograms of $A_{K}, 2 \mathrm{MASS}$ values for all the cells in the W0.2-2.1 and W359.4-3.1 maps. In W0.2-2.1 there is a larger number of cells with high $A_{K}$, 2MASs values than in W359.4-3.1. This reflects the closer proximity of the former field to the Galactic Centre. Panels b) and d) of the same figure show the internal errors in the determination of the $A_{K}, 2 \mathrm{MASS}$ values as a function of $A_{K}, 2 \mathrm{MASS}$ itself. These errors correspond to the std of the final, sigma-clipped, histogram of $A_{K}, 2 \mathrm{MASS}$ values in each cell. We notice that in W0.2-2.1 the internal errors have a larger dispersion with $A_{K}, 2$ MAss. Such behaviour was also found by FTK99 and is due to the small scale variations of the dust distribution in the region. The mean internal errors are $\langle\sigma\rangle=$ $0.09 \pm 0.03$ in $\mathrm{W} 0.2-2.1$ and $\langle\sigma\rangle=0.08 \pm 0.03$ in

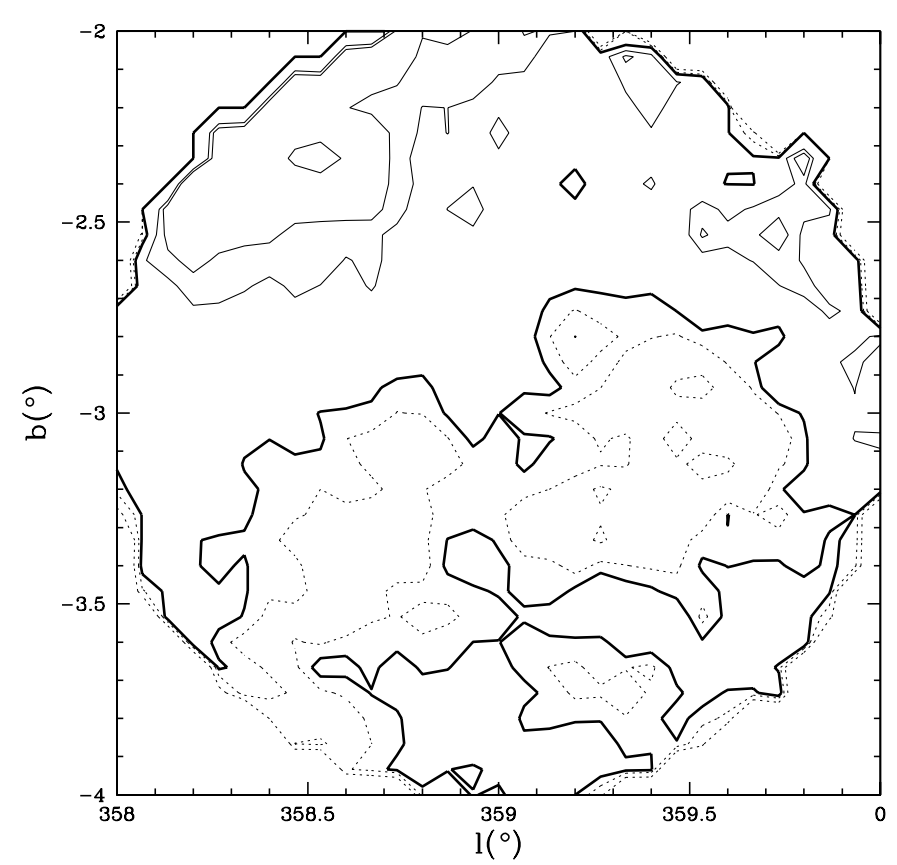

Fig. 7. 2MASS extinction map of window W359.4-3.1. Contour levels correspond to $A_{K}, 2 \mathrm{MASS}=0.2$ and 0.25 (dotted lines), 0.28 (thick solid lines), and 0.4 and 0.5 (thin solid lines).
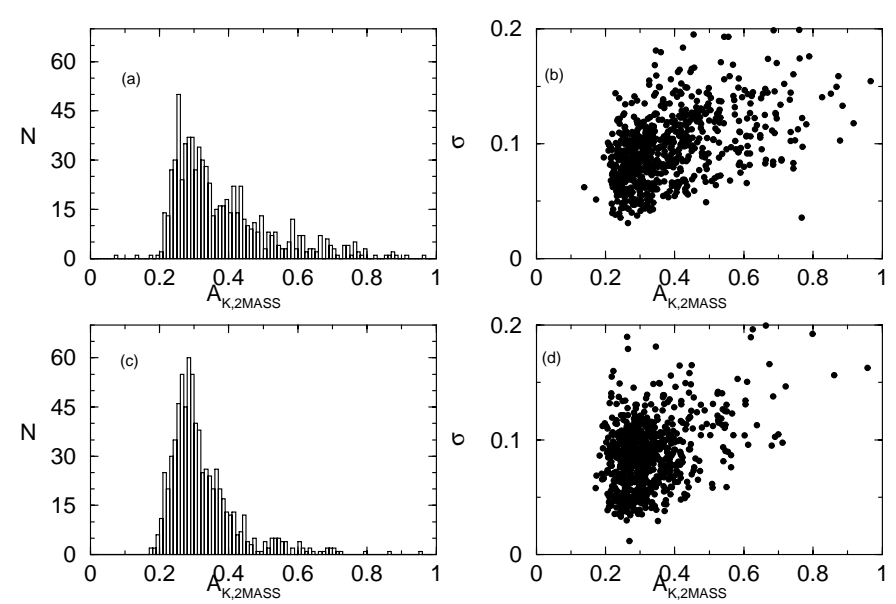

Fig. 8. 2MASS extinction histogram for window W0.2-2.1 in panel a), and for W359.4-3.1 in panel c). Extinction internal errors as a function of $A_{K}$, 2MASS for W0.2-2.1 in panel b), and for W359.4-3.1 in panel $\mathbf{d}$ ).

W359.4-3.1. These errors are somewhat higher than those estimated by FTK99 in their Bulge fields. The reason is that that our $4^{\prime} \times 4^{\prime}$ cells are larger than those used by FTK99 $\left(1.5^{\prime} \times 1.5^{\prime}\right)$ and should, therefore, include a larger dispersion by dust gradients.

The bulge windows W0.2-2.1 and W359.4-3.1 are relatively closer to the Galactic Centre than Sgr I, Sgr II and Baade's windows and are located in a hole surrounded by the dark clouds LDN48, LND43, LDN1801, LDN1769, LDN1783, LDN3, LDN1795, LDN1788 from the Lynds' (1962) catalogue and FSDN435, FSDN431, FSDN430, FSDN444 from the Feitzinger \& Stüwe's (1984) catalogue. 

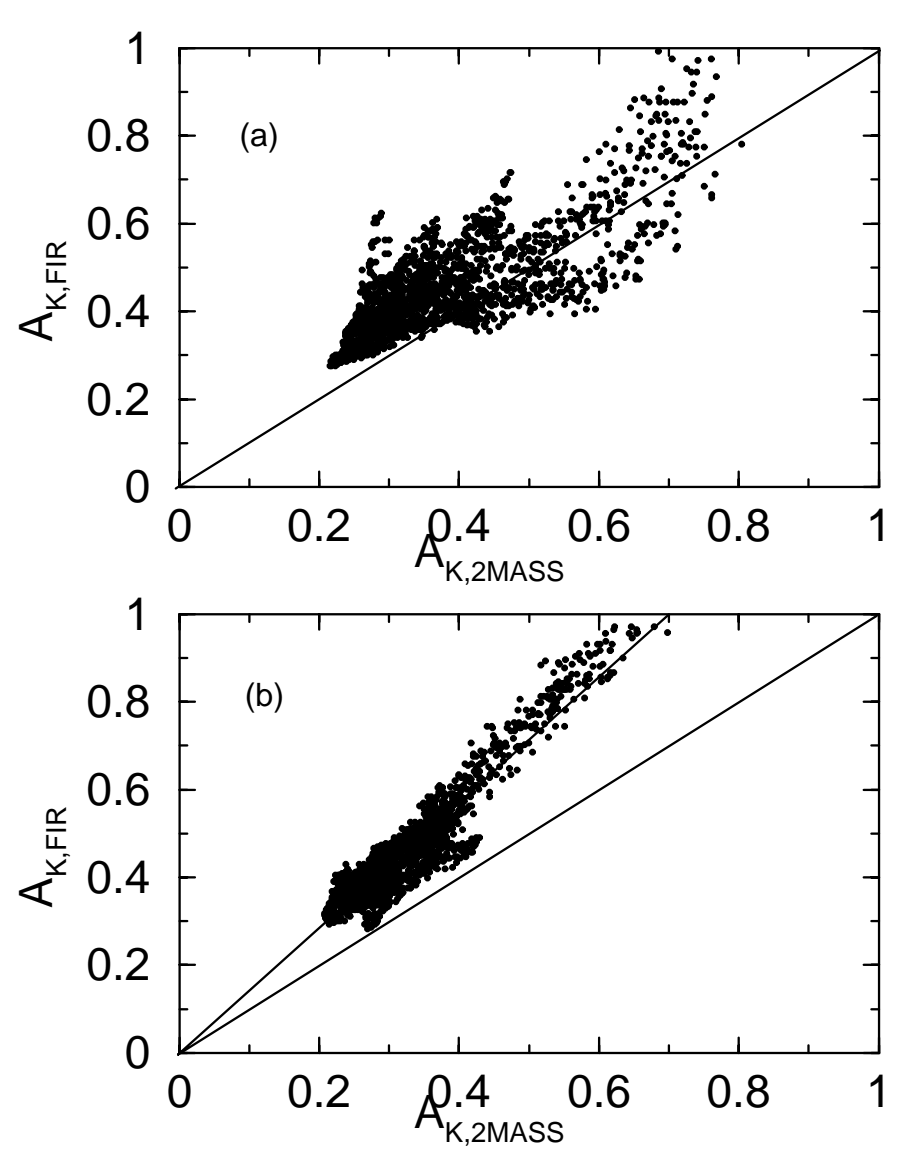

Fig. 9. Comparison of FIR and 2MASS extinction values for cells in windows W0.2-2.1 (panel a)) and W359.4-3.1 (panel b)). The identity function is indicated as a straight line. The function $A_{K}$, FIR $=1.45 A_{K}$, 2MASS is also indicated in panel b).

\subsection{Comparison between 2 MASS and FIR emission extinction maps}

As mentioned previously, the 2MASS extinction maps of Figs. 6 and 7 show essentially the same features as the FIR extinction maps (Figs. 2 and 3). In order to investigate this similarity in a more quantitative manner, we convolve the 2MASS $A_{K}$ extinction maps with a $\sigma=4.5^{\prime}$ Gaussian, obtaining a resolution of approximately $6^{\prime} F W H M$, which is compatible with that of SFD98's extinction maps $\left(6.1^{\prime}\right)$. Figure 9 shows the comparison between the $A_{K}$, 2MASS and $A_{K}$, FIR values for both low-extinction windows.

In both panels we see a clear correlation between $A_{K}$, FIR and $A_{K}$, MASS, quantitatively confirming the similarities between the two extinction maps. The relation between the two $A_{K}$ estimates, however, has a much more complex pattern in W0.2-2.1 (Panel 9a) than in W359.43.1 (Panel 9b). We see several branches in panel 9a, some above the identity line, some lying below it. We verify that the cells that form these particular structures in the $A_{K}$ scatter plot correspond to specific spatial regions in the extinction map. These may correspond to lines of sight crossing specific dust clouds whose physical characteristics, most specially dust temperature and density, could yield distinct signatures in their emission and absorption properties. As mentioned in the end of the previous section, several individual dark clouds lying close to and around our two low-extinction regions have been catalogued.

In the W359.4-3.1 region (panel 9b), there is a distribution much closer to linear relation between $A_{K}$, 2MASS and $A_{K, \text { FIR }}$. The slope in this relation, however, is larger than unity: $A_{K}$, FIR $=1.45 A_{K}$, 2MASS. Arce \& Goodman (1999) obtained a similar result in their analysis of the Taurus dark cloud complex $\left(b \approx-15^{\circ}\right)$; by comparing the dust emission values derived by SFD98 to those derived from four other methods, including a dust emission extinction derived directly from $100 \mu \mathrm{m}$ flux with temperature corrections. They concluded that SFD98 may be overestimating extinction by a factor varying from 1.3 to 1.5 . They also pointed out that this factor could not be due to overestimation of the ratio of total-to-selective extinction $R_{V}$, because several studies (Kenyon et al. 1994; Vrba \& Rydgren 1985; and recently Whittet et al. 2001) suggested that the $R_{V}=3.1$ in most parts of the Taurus dark cloud complex, in regions where the visual extinction is lower than $A_{V}<3.0$. This systematic effect in $A_{K}$, FIR may originate from SFD98's calibration of the dust column density $\times$ reddening relation, which was derived in regions of low to moderate extinction $(E(B-V) \simeq 0.15)$, but may not apply equally well to the high extinction regime.

As a helping tool in understanding the differences in the $A_{K}$, FIR and $A_{K}$, 2MASs values, we have considered the amount of dust expected to lie beyond the inner Galactic Bulge, therefore being located behind most of the stars measured by 2MASS. This dust located on the far side of the Galaxy will have an obvious influence over the $A_{K}$, FIR $\times A_{K}$, 2MASS correlation, since it is expected to affect the first but not the second extinction value. A simple model of dust distributed on a plane with an exponential drop both along and perpendicular to this plane has been used to predict the relative contribution of the material on the far side of the Galaxy. We thus consider a model of linear absorption coefficient given by:

$\sigma_{\lambda} \propto \mathrm{e}^{-R / R_{\mathrm{d}}} \mathrm{e}^{-Z / Z_{\mathrm{d}}}$

where $R$ and $Z$ are cylindrical coordinates centred on the Galaxy, whereas $R_{\mathrm{d}}$ and $Z_{\mathrm{d}}$ are the dust horizontal and vertical exponential scales, respectively. By adopting reasonable values for the scale parameters (such as $R_{\mathrm{d}} \simeq 2.5 \mathrm{kpc}$ and $Z_{\mathrm{d}} \simeq 100 \mathrm{pc}$ ) and integrating the dust absorption along the two lines of sight where our lowextinction windows are located, we conclude that $<20 \%$ of the total extinction towards W0.2-2.1 is caused by dust on the background of the stars belonging to the inner Bulge in this direction. For W359.4-3.1, this amount is $5 \%$ or less. Therefore, we conclude that dust on the far side of the Galaxy may partially account for the differences in the DIRBE/IRAS and 2MASS $A_{K}$ determinations, especially for $A_{K}$, FIR being larger than $A_{K, 2 \text { MASS }}$ in most cells. On the other hand, background dust does not explain the 


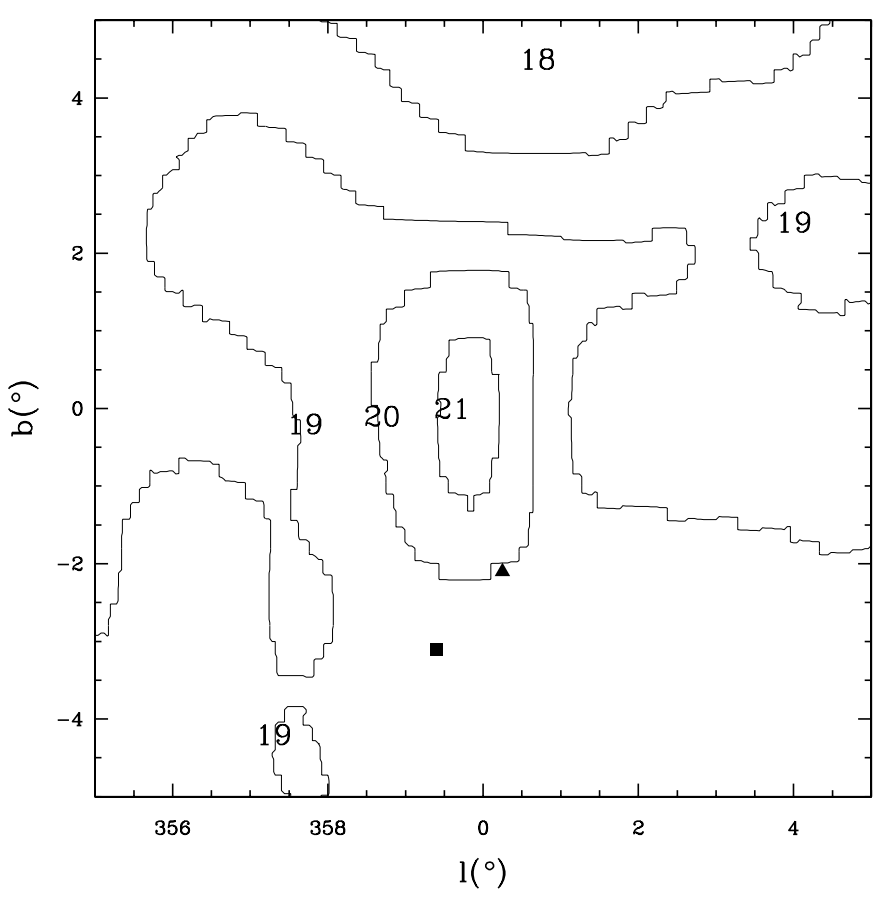

Fig. 10. $10^{\circ} \times 10^{\circ}$ temperature map centred on the Galactic Nucleus, according to Schlegel et al. (1998). The temperature contours are given in Kelvin. The triangle and square correspond to the position of W0.2-2.1 and W359.4-3.1, respectively.

complex patterns seen in W0.2-2.1 or the slope in the W359.4-3.1 scatter plot.

Temperature effects may play a role in explaining the observed features, since dust clouds in the direction of the Galactic Centre may be warmer on average than elsewhere. In addition, the low resolution $\left(\approx 1^{\circ}\right)$ of SFD98's temperature maps increases the uncertainties in the $A_{K}$, FIR temperature corrections, especially in zones of large temperature gradients. Figure 10 shows SFD98's temperature map in the central $10^{\circ}$ of the Galaxy. Despite the low resolution, we notice that W0.2-2.1 is much closer to a local peak in the dust temperature map than W359.43.1. This adds support to the idea of temperature gradients contributing to the complexity of Panel 9a as compared to Panel $9 \mathrm{~b}$.

Additional sources of scatter in the $A_{K}, 2$ MASS $\times$ $A_{K}$, FIR relation are possible contamination effects in both datasets. As pointed out by SFD98, extragalactic and unresolved Galactic sources at low Galactic latitudes $(|b|<$ $5^{\circ}$ ) have not been removed from the dust maps. They could be an important contribution to increase $A_{K}$, FIR values in very low latitudes, in particular in the Bulge window closer the Galactic Plane, W0.2-2.1. Concerning the 2MASS data, apart from applying the $\sigma$ clipping of the $A_{K}$ distribution in each cell, we did not attempt any further correction for contaminating foreground disk stars.

\section{Concluding remarks}

We identified a new low-extinction window towards the Galactic Bulge, W359.4-3.1, located at $(\ell, b)=\left(359.40^{\circ}\right.$, $-3.10^{\circ}$ ) and of size $40^{\prime} \times 30^{\prime}$, using a $10^{\circ} \times 10^{\circ}$ map around the Galactic Centre extracted from Schlegel et al.'s (1998) FIR emission reddening database.

Using the infrared photometry available from the 2MASS $J H K_{\mathrm{s}}$ survey archive, we built $A_{K}$, 2MASS extinction maps for W359.4-3.1 and for W0.2-2.1, this latter window previously identified by Stanek (1998). The extinction values were determined by means of upper giant branch fitting, using as a reference the upper giant branch of fields previously studied by Frogel et al. (1999). The extinction determination method and the 2MASS photometry were tested on fields with well known low-extinction values: Sgr I and Baade's Window (Baade 1963). Our derived extinction values for these windows agree well with previous values quoted in the literature, indicating that the present method is a useful tool for the study of the bulge windows.

The $A_{K}$, 2MAss maps confirmed the existence of the two bulge windows. The mean extinction in the field around W0.2-2.1 is $\left\langle A_{K}\right.$, 2MASS $\rangle=0.29 \pm 0.05$, whereas in the field around W359.4-3.1 we obtained $<A_{K, \text { 2MASS }}>=0.28 \pm 0.04$. In both cases we find an area of systematically lower than average $A_{K}$, 2MASS values, with minima around $A_{K}, 2$ MAss $\simeq 0.20$. These windows are located very close to the Galactic Centre, through a hole in the distribution of known dark clouds. The extinction maps obtained with 2MASS data show very similar features to those based on FIR dust emission data. However, some systematic effects, usually in the sense that $A_{K}$, FIR $>A_{K}, 2 \mathrm{MASS}$, are seen when a direct comparison of the two extinction estimates is made. In particular, for the W359.4-3.1 region, $A_{K}$, FIR $\simeq 1.45 \times$ $A_{K}, 2$ MAss with a small scatter. For W0.2-2.1, which lies closer to the Galactic Centre, the situation is more complex, with several structures with distinct correlations between dust emission and absorption being present.

The qualitative agreement suggests that the dust clouds that redden the bulk of Bulge stars are the main contributors to the dust emission in the region. Indeed, a simple model for the distribution of dust shows that most of the dust clouds in the line of sight towards the lowextinction windows should be located on the near side of the Galaxy. The amount of dust expected to lie beyond the Galactic Centre does not alone explain the quantitative differences between $A_{K}$, FIR and $A_{K}$, 2MASs. These may be caused by temperature variations as a function of distance from the centre of the Galaxy or from one individual dust cloud to another. Alternatively, systematic effects in the conversion of DIRBE/IRAS maps into extinction measures might explain the observed differences. It is not possible to disentangle these effects using only the data shown here.

These low-extinction windows are interesting new targets to studies of the properties of the Bulge stellar 
population, especially considering their location closer to the Galactic Centre than Baade's Window.

Acknowledgements. We thank Dr. D. Schlegel for pointing out to us the availability of a new version of his software dust-getval for reading the temperature maps. This publication makes use of data products from the Two Micron All Sky Survey, which is a joint project of the University of Massachusetts and the Infrared Processing and Analysis Center/California Institute of Technology, funded by the National Aeronautics and Space Administration and the National Science Foundation. We also have made use of the NASA/IPAC Infrared Science Archive, which is operated by the Jet Propulsion Laboratory, California Institute of Technology, under contract with the NASA. We acknowledge support from the Brazilian institutions FAPESP and CNPq. CD acknowledges the FAPESP pos-doc fellowship Proc. 00/11864-6.

\section{References}

Aguerri, J. A. L., Balcells, M., \& Peletier, R. F. 2001, A\&A, 367,428

Alard, C., Blommaert, J. A., Cesarsky, C., et al. 2001, ApJ, 552,289

Alcock, C., Allsman, R. A., Alves, D. R., et al. 1998, ApJ, 494, 396

Arce, H. G., \& Goodman, A. A. 1999, ApJ, 512, 135

Baade, W. 1963, Evolution of stars and galaxies (Harvard University Press, Cambridge, Mass), 277

Cardelli, J. A., Clayton, G. C., \& Mathis, J. S. 1989, ApJ, 345, 245

Dutra, C. M., \& Bica, E. 2000, A\&A, 359, 347

Epchtein, N., de Batz, B., Capoani, L., et al. 1997, The Messenger, 87, 27

Feitzinger, J. V., \& Stüwe, J. A. 1984, A\&AS, 58, 365

Frogel, J. A., Tiede, G. P., \& Kuchinski, L. E. 1999, AJ, 117, 2296 (FTK99)
Glass, I. S., Whitelock, P. A., Catchpole, R. M., \& Feast, M. W. 1995, MNRAS, 273, 383

Gould, A., Popowski, P., \& Terndrup, D. M. 1988, ApJ, 492, 778

Hammersley, P. L., Garzon, F., Mahony, T., \& Calbet, X. 1995, MNRAS, 273, 206

Kenyon, S. J., Dobrzycka, D., \& Hartman, L. 1994, AJ, 108 , 1872

Lloyd Evans, T. 1976, MNRAS, 174, 169

Lynds, B. T. 1962, ApJS, 7, 1

Mathis, J. S. 1990, ARA\&A, 28, 37

Persson, S. E., Murphy, D. C., Krzeminski, W., Roth, M., \& Rieke, M. J. 1998, AJ, 116, 2475

Ramírez, S. V., Stephens, A. W., Frogel, J. A., \& DePoy, D. L. 2000, AJ, 120, 833

Schlegel, D. J., Finkbeiner, D. P., \& Davis, M. 1998, ApJ, 500, 525 (SFD98)

Schulteis, M., Ganesh, S., Simon, G., et al. 1999, A\&A, 349, L69

Skrutskie, M., Schneider, S. E., Stiening, R., et al. 1997, in The Impact of Large Scale Near-IR Sky Surveys, ed. F. Garzon et al. (Kluwer, Netherlands), 210, 187

Stanek, K. Z. 1996, AJ, 460, L37

Stanek, K. Z. 1998, Using the DIRBE/IRAS all-sky reddening map to select low-reddening windows near the Galactic Plane [astroph/9802307]

Terndrup, D. M. 1988, AJ, 96, 884

Tiede, G. P., Frogel, J. A., \& Terndrup, D. M. 1995, AJ, 110, 2788

Unavane, M., Gilmore, G., Epchtein, N., et al. 1998, MNRAS, 295,119

Vrba, F. J., \& Rydberg, A. 1985, AJ, 90, 1490

Wyse, R. F. G., Gilmore, G., Norries, J. E., \& Freeman, K. C. 2000, AAS, 197, 424

Whitford, A. E. 1978, ApJ, 226, 777

Whittet, D. C. B., Gerakines, P. A., Hough, J. H., \& Shenoy, S. S. 2001, ApJ, 547, 872 